\title{
La noción de "Competencias" en la Reforma de la Educación Básica de México (2009-2011): Limitaciones y alternativas
}

JUAN CARLOS MIRANDA ARROYO

Universidad Pedagógica Nacional, Unidad Querétaro, México.

"El análisis de competencias remite constantemente a una teoría del pensamiento y de la acción situados

(Gervais, 1998); pero también del trabajo, la práctica como profesión y condición (Descolonges, 1997;

Perrenoud, 1996c). Es decir, que nos hallamos en terreno pantanoso, a la vez que en el plano de conceptos e ideologías...".

Philippe Perrenoud. Diez nuevas competencias para enseñar. Biblioteca para la Actualización del Maestro México, SEP, 2004. p. 11.

\section{Introducción}

De acuerdo con el reporte de la Organización para la Cooperación y el Desarrollo Económico IOCDE, 2001) denominado: "La Definición y selección de Competencias Clave", "...una competencia es más que conocimientos y destrezas. Involucra la habilidad de enfrentar demandas complejas, apoyándose en y movilizando recursos psicosociales (incluyendo destrezas y actitudes) en un contexto en particular. Por ejemplo, la habilidad de comunicarse efectivamente es una competencia que se puede apoyar en el conocimiento ("que posee o domina") un individuo del lenguaje, destrezas prácticas en tecnología e información, y actitudes con las personas que se comunica".

Esta caracterización que publicó en 2001 la OCDE sobre las "competencias educativas", ha tenido repercusiones importantes en el rediseño de planes y programas educativos durante los últimos años en países miembros de ese organismo. Y quizá también constituye una aportación a favor de la necesidad de aclarar ese término ("competencias educativas"), en el sentido "integrador" que el concepto demanda; por lo tanto, el equipo técnico de la OCDE habla de "competencias" dentro de un perfil menos fragmentario que aquellas versiones curriculares de la posguerra. Todo lo contrario, por ejemplo, de la lógica que llevaba implícita la taxonomía de los "objetivos educacionales" de Benjamín Bloom (1957) o de otras aproximaciones teóricas y metodológicas que se han especializado en el diseño, análisis y evaluación de programas educativos (como son los casos de J. Araujo y C. Chadwick, 1988; de R. H. Davis y cols., 1988; de Robert Gagné, 1977 o de R. Mager, 1970 y 1975, entre otros).

Es importante considerar que el modelo de planeación curricular, en general, y de planeación didáctica y diseño de ambientes de aprendizaje, en particular, que está implícito en el reporte de la OCDE, y

\footnotetext{
'Resumen ejecutivo preparado por el grupo educativo de la Organización para la Cooperación y el Desarrollo Económico (OCDE, 2001; "OECD" por sus siglas en inglésl, y traducido con fondos de la Agencia de los Estados Unidos para el Desarrollo Internacional (USAID).
}

Revista Iberoamericana de Educación / Revista Ibero-americana de Educação

ISSN: $1681-5653$

n. ${ }^{\circ} 61 / 4-15 / 04 / 13$

Organización de Estados Iberoamericanos para la Educación, la Ciencia y la Cultura (OEI-CAEU)

Organização dos Estados Ibero-americanos para a Educação, a Ciência e a Cultura (OEI-CAEU) 
cuyo fundamento teórico es el enfoque psicopedagógico "basado en el desarrollo de competencias", parece ser un modelo renovado de la taxonomía de los llamados "paquetes de aprendizaje", sin embargo, no es el único ni el más completo de los modelos existentes en la literatura internacional, desde la perspectiva de la investigación educativa en materia de diseño curricular. Aunque, -cabe decirlo claramente-, a este modelo se le reconoce, hoy en día, como uno de los más influyentes en el diseño de los programas indicativos internacionales en el campo educativo, asi como en el trazo de las políticas públicas educativas regionales o nacionales de todos los niveles y modalidades: desde posgrado hasta preescolar.

Pero esta renovación del modelo curricular no necesariamente ha impactado favorablemente en la cultura pedagógica, ni en las prácticas docentes dentro de las escuelas de las naciones que lo han adoptado, como decisión centralizada de los gobiernos nacionales o federales; sobre todo en aquellas sociedades donde se han iniciado procesos de reforma curricular de la educación formal o escolarizada (superior, media y básica, en ese orden) desde el año 1990, aproximadamente, a la fecha, tal como es el caso de México (SEP, 2009 y $2011^{3}$ ).

Dicho enfoque presenta, sin embargo, algunas limitaciones o inconsistencias en su núcleo argumentativo ${ }^{4}$, mismas que conviene revisar con una actitud crítica:

- La herencia marcada desde su creación ("la falla de origen"), ya que ese enfoque se deriva de la definición de "perfiles profesionales de desempeño": hay que recordar que los perfiles laborales de las profesiones pasaron por varias etapas o cambios; desde el diseño de puestos basado en o "por objetivos", al diseño centrado en propósitos, funciones, responsabilidades y, finalmente, por el desarrollo de "competencias".

- En segundo lugar, el enfoque aludido está supeditado a la lógica de la "competencia social" como conjunto de conductas o comportamientos que pueden ser medidos o evaluados, a través de un estándar de desempeño o norma, y no necesariamente como un expresión de la actividad humana en términos del desarrollo integral de las personas. Por lo anterior, el enfoque educativo "orientado hacia el desarrollo de competencias" tiene la desventaja de quedar limitado por criterios pragmáticos, porque hace énfasis en los resultados acerca de lo que "sabe hacer" el sujeto evaluado y no tanto en los procesos educativos o en las acciones específicas que conducen a la obtención de dichos resultados (una evaluación superficial de los aprendizajes fundado con este enfoque diría lo siguiente con respecto al sujeto: "lo sabe o no lo sabe hacer...").

\footnotetext{
${ }^{2}$ La noción de "paquetes de aprendizaje" se desarrolló durante los años 70s del siglo XX, y se refiere a la integración de los distintos campos o áreas de aprendizaje durante los procesos de planeación didáctica, (para ver más información al respecto, sugiero la lectura del libro de Robert Gagné: "Las condiciones del aprendizaie", (1977), donde el autor aborda los conceptos de: "habilidades intelectuales", habilidades psicomotrices", "información verbal", "estrategias cognoscitivas" y aprendizaje de "actitudes y valores", que perfeccionaron los dominios o áreas de la taxonomía de B. Blooml.

${ }^{3}$ El Programa Sectorial de Educación 2007-2012 de México, así lo asume en su objetivo 1. "Elevar la calidad de la educación para que los estudiantes mejoren su nivel de logro educativo, cuenten con medios para tener acceso a un mayor bienestar y contribuyan al desarrollo nacional", bajo el rubro de Educación Básica, estrategia 1.l, señala la necesidad de "realizar una Reforma Integral de la Educación Básica, centrada en la adopción de un modelo educativo basado en competencias, que responda a las necesidades de desarrollo de México en el siglo XXI, estableciendo, entre otras líneas de acción, la de asegurar que los planes y programas de estudio estén dirigidos al desarrollo de competencias e involucrar activamente a los docentes frente a grupo en estos procesos de revisión y adecuación, y que esta acción tendrá como base los resultados de las evaluaciones del logro educativo, así como la de establecer estándares y metas de desempeño en términos de logros de aprendizaje esperados en todos sus grados, niveles y modalidades".

${ }^{4}$ César Coll y Elena Martín (2006). Vigencia del debate curricular. Aprendizajes básicos, competencias y estándares. II Reunión del Comité Intergubernamental del Proyecto Regional de Educación para América Latina y el Caribe (PRELAC). UNESCO-OREALC. Oficina Regional de Educación para América Latina y el Caribe. Santiago de Chile.
} 
Con ello se sacrifica la riqueza del amplio espectro de "acciones intelectuales" Icomo, por ejemplo, de reflexión teórica, filosófica, ética, etc.l, o bien del ámbito de las habilidades "psicomotrices" o "psicoemocionales", por poner algunos otros ejemplos que tienen lugar en la vida cotidiana de la educación escolarizada, y sobre todo cuando esto se da en la vida cotidiana de la Educación Básica (Preescolar, Primaria y Secundaria), que sólo se justifica a cambio de favorecer la generación de respuestas concretas a evaluaciones "objetivas". En esa línea de ideas, los promotores del enfoque en cuestión, Idenominado: "de desarrollo de competencias"), consideran que las personas son "competentes" al término de un proceso educativo porque "dan resultados" ante situaciones específicas (por ejemplo, un examen escritol, o porque pueden ser evaluadas o pueden demostrar sus conocimientos, habilidades o valores en términos de los "desempeños empíricamente verificables". ${ }^{5}$ Otra crítica interesante en este sentido es la que nos sugiere César Coll sobre los riesgos de tomar al concepto de "Competencias educativas" en sentidos extremos: como una moda o como un remedio. ${ }^{6}$

- Existe también una tercera observación crítica: un intento de aplicación, de manera transferida del concepto "competencias": de la Educación Superior a la Educación Básica: Si bien la planeación curricular a partir del diseño basado "en el desarrollo de competencias" tiene en cierto grado de éxito en la educación superior, dada la cercanía que ésta tiene con los perfiles de egreso de los futuros profesionistas (recuérdese la creación del concepto: "perfiles profesionales de desempeño" en las carreras universitarias o en los programas de posgradol, en la Educación Básica, y eventualmente en la educación media superior, eso no ocurre o al menos la posibilidad de éxito es más remota, debido a la lejanía observada en la aplicación de los procesos y los resultados de los aprendizajes escolares, conforme a ciertos perfiles de egreso. Por esa razón la SEP (2009) ha publicado un perfil de egreso para la Educación Básica en general y ha logrado apenas definir estándares curriculares y aprendizajes esperados (2011), por nivel educativo: para la Educación Preescolar, Primaria y Secundaria)?

- Una última reflexión crítica en torno al concepto de "competencias" en contextos educativos, tiene estrecha relación con lo expuesto por Coll y Martín (Op. cit.), cuando exponen la siguiente pregunta:

"¿Cuáles son los aprendizajes que todo el alumnado debería poder alcanzar en el transcurso de la educación básica?". A lo cual ellos mismos responden: "Desde hace ya algunos años el debate sobre los aprendizajes básicos refleja cada vez con mayor intensidad la tensión generada por la necesidad de atender a dos exigencias que parecen orientarse en direcciones opuestas. Por una parte, en el nuevo escenario social,

\footnotetext{
5 Xabier Garagorri en su artículo "Currículo basado en competencias: aproximación al estado de la cuestión" (Revista Innovación Educativa, No. 1611, señala lo siguiente: "Hay una serie de conocimientos, de experiencias y de actitudes que hacen que seamos quienes somos, que son importantes para nuestra vida y que no desembocan forzosamente en competencias para resolver problemas, sino que sirven para comprendernos a nosotros mismos, a la sociedad o a la naturaleza. Conocimientos relacionados con la filosofía, la psicología, la antropología, la historia, la física, etc. Por ejemplo, Edgar Morin (2000) propone que hay siete saberes que considera fundamentales para la Educación: 1. Conocer lo que es conocer y evitar las cegueras del error y la ilusión. 2. Conocer los principios del conocimiento pertinente. 3. Enseñar la condición humana: reconocerse en los elementos comunes de la identidad de la especie humana y al mismo tiempo reconocer la diversidad tanto individual como cultural. 4. Tomar conciencia de la identidad terrenal. 5. Saber afrontar las incertidumbres. 6. Saber comprender. 7. Conducirse de acuerdo con la ética del género humano. Algunos de estos saberes tienen sentido en la medida en que se traducen en competencias para conocer, comprender, afrontar incertidumbres o conducirse de acuerdo con la ética, pero hay otros saberes que conforman nuestro pensamiento, memoria e identidad (en definitiva, nuestra forma de serl, que están más próximos del polo del pensamiento que de la acción y que tienen valor por sí mismos, independientemente de que pensamiento y acción se necesiten mutuamente".

${ }^{6}$ César Coll, Las competencias en la educación escolar: algo más que una moda y mucho menos que un remedio. Revista Innovación Educativa, No. 161.

7 Documento: SEP. ACUERDO número 592 por el que se establece la Articulación de la Educación Básica. México. Diario Oficial de la Federación. 19 de agosto de 2011.
} 
económico, político y cultural que están contribuyendo a dibujar los movimientos migratorios, los procesos de globalización, las tecnologías digitales de la información y la comunicación, la economía basada en el conocimiento, etc., parece cada vez más evidente la necesidad de incorporar nuevos contenidos al curriculum de la educación básica. La convicción de que algunas competencias y contenidos de aprendizaje esenciales para el ejercicio de la ciudadanía en este nuevo escenario se encuentran escasamente representadas en el curriculum escolar está ampliamente extendida y se encuentra en la base de una demanda generalizada para subsanar con urgencia esta carencia. Esta demanda se ve además reforzada como consecuencia de la creciente "des-responsabilización social y comunitaria" ante la educación (Coll, 2003) que ha llevado a transferir a la educación escolar la responsabilidad de unos aprendizajes que hasta épocas recientes era asumida por otras instancias educativas, de socialización y de formación (familia, iglesia, agrupaciones políticas y sindicales, asociaciones diversas, etc.)".

"Por otra parte, sin embargo, en muchos países amplios sectores del profesorado de la educación básica coinciden en valorar, y nosotros compartimos esa valoración, que es más bien imposible que el alumnado pueda aprender y el profesorado pueda enseñar todos los contenidos ya incluidos en los currícula vigentes. Se trata también de una valoración extendida, pero que conduce en este caso a subrayar la necesidad de una revisión del curriculum en una dirección opuesta a la anterior, es decir, orientada más bien a reducir los contenidos de aprendizaje. En efecto, las implicaciones altamente negativas para la calidad de la educación escolar de unos curricula sobrecargados y excesivos son de sobra conocidas" ${ }^{\prime 8}$ (subrayados de J. C. M.A.).

Los investigadores españoles concluyen asi su crítica: "...en estos niveles educativos (preescolar, primaria y secundaria) no se puede enseñar todo lo que nos gustaría que los niños y jóvenes aprendiesen; ni siquiera lo que con toda seguridad es beneficioso que los niños y jóvenes aprendan"... "Hay que hacer opciones. Hay que elegir. Cuando se amplían o se introducen nuevos contenidos o nuevas competencias en el curriculum de la educación básica, hay que recortar o excluir otros. Ni el curriculum ni el horario escolar son como chicle o una goma elástica. Los curricula sobrecargados que no tienen en cuenta este hecho son un obstáculo para el aprendizaje significativo y funcional, una fuente de frustración para el profesorado y el alumnado y una dificultad añadida para seguir avanzando hacia una educación inclusiva".

"... La toma en consideración de este principio y de sus implicaciones sugiere la conveniencia de explorar, y en su caso establecer, una distinción entre lo básico imprescindible y la básico deseable en el curriculum de la educación básica".

"El término "básico" es utilizado habitualmente, en el marco del curriculum escolar y referido a la concreción de las intenciones educativas -aprendizajes esperados del alumnado definidos en términos de competencias o de contenidos de aprendizaje-, con una multiplicidad de significados interconectados e interrelacionados. Los contenidos y competencias identificados como básicos con el fin de justificar su presencia en el curriculum escolar remiten siempre a la realización de unos aprendizajes considerados necesarios para los alumnos. La polisemia del concepto reside no tanto en la supuesta necesidad de los aprendizajes, como en la finalidad o propósito para cuya consecución dichos aprendizajes se consideran necesarios. Así, es habitual que la presencia de los contenidos o competencias en el curriculum de la educación básica se justifique argumentando que su aprendizaje es necesario para alcanzar uno o varios de los propósitos siguientes : a) para hacer posible el pleno ejercicio de la ciudadanía en el marco de la sociedad de referencia; bl para poder construir y desarrollar un proyecto de vida satisfactorio; cl para asegurar un desarrollo personal emocional y afectivo equilibrado; o d) para poder acceder a otros procesos educativos y formativos posteriores con garantías de éxito"'"

Por lo anterior, es conveniente guardar distancia en torno a ese enfoque y no caer en el triunfalismo al afirmar que hoy "la educación moderna tiene su fortaleza en el diseño curricular orientado al desarrollo de competencias". Nada más alejado de la realidad. Y doble falta se tiende a cometer cuando los nuevos plan y

\footnotetext{
${ }^{8}$ C. Coll y E. Martín, Op. cit.. p. 5

${ }^{9}$ C. Coll y E. Martín, Op. cit., p. 7
} 
programas de la Educación Básica (2011), no sólo se han diseñado a partir de la noción de "desarrollo de competencias" (Preescolar en 2004, Secundaria en 2006 y Primaria en 2009), sino que se han elevado a rango de normatividad institucional (Acuerdo 592 de la SEP). Hoy en México la Educación Básica fundada en la idea de desarrollar las "competencias educativas" no sólo constituye una propuesta técnica, sino un ideal y una normatividad que, por tanto, se ha convertido en Acuerdo o Reglamento para docentes, asesores académicos y directivos: los maestros y las maestras deben pensar y actuar conforme al modelo decretado y legitimado, a través de métodos o procedimientos centralizados ${ }^{10}$, en el marco de un currículo escolar único, nacional y obligatorio.

Si bien es importante considerar la normatividad actualmente aprobada por la SEP (diseño curricular "basado en el desarrollo de competencias educativas"), es también relevante tomar en cuenta todos aquellos enfoques teóricos y metodológicos de la planeación curricular ly de su expresión operativa: la planeación didáctica), que den cuenta de criterios menos limitados y más ajustados a la realidad de la Educación Básica.

Es importante para ello considerar, en primer lugar, la definición de las "competencias clave" que elaboró o definió la OCDE, y posteriormente describir y analizar las competencias educativas definidas por la Secretaría de Educación Pública (SEP, México), en términos del perfil general de egreso de la Educación Básica.

- Descripción de las "competencias clave" (OCDE) y "perfil de egreso" de la Educación Básica en México (SEP)

A continuación se describirán, en dos secciones, los siguientes puntos:

$\checkmark$ Las llamadas "Competencias Clave" que fueron definidas por la OCDE a finales de la década de los 90־s e inicio del 2000, y b) el "perfil de egreso" de la Educación Básica, publicado por la SEP durante la década que recién concluyó $(2000,2004,2006$ y 2009).

$\checkmark$ Competencias Clave (Organización para la Cooperación y el Desarrollo Económico, OCDE): "Competencias clave" en tres amplias categorías:

"El marco conceptual del proyecto denominado por la OCDE "Desarrollo de Competencias Clave" (DeSeCo), clasifica dichas competencias en tres amplias categorías. Primero, los individuos deben usar un amplio rango de herramientas para interactuar efectivamente con el ambiente: tanto físicas como en la tecnología de la información y socio culturales como en el uso del lenguaje. Necesitan comprender dichas herramientas ampliamente, cómo para adaptarlas a sus propios fines, usar las herramientas de manera interactiva. Segundo, en un mundo cada vez más interdependiente, los individuos necesitan poder comunicarse con otros, y debido a que encontrarán personas de diversos orígenes, es importante que puedan interactuar en grupos heterogéneos. Tercero, los individuos necesitan poder tomar la responsabilidad de manejar sus propias vidas, situar sus vidas en un contexto social más amplio y actuar de manera autónoma' (p. 4).

\begin{tabular}{|c|c|c|}
\hline \multicolumn{2}{|c|}{ Competencias clave } \\
\hline $\begin{array}{c}\text { 1. Usar herramientas de manera } \\
\text { interactiva lejs.: lenguajes, tecnología) }\end{array}$ & 2. Interactuar en grupos heterogéneos & 3. Actuar de forma autónoma \\
\hline $\begin{array}{c}\text { Competencia l-a: } \\
\text { La habilidad para usar el lenguaje, los } \\
\text { simbolos y el texto de forma interactiva }\end{array}$ & $\begin{array}{c}\text { Competencia 2-a: } \\
\text { La habilidad de relacionarse bien con otros }\end{array}$ & $\begin{array}{c}\text { Competencia 3-a: } \\
\text { Labilidad de actuar dentro del } \\
\text { gran esquema o panorama }\end{array}$ \\
\hline
\end{tabular}

\footnotetext{
${ }^{10}$ Acción No. V del proceso de elaboración del currículo: "Se construyeron consensos sociales sobre el currículo, que fueron resultado del trabajo de la Secretaría de Educación Pública del Gobierno Federal con las autoridades educativas locales, el Sindicato Nacional de Trabajadores de la Educación, así como con diversas instituciones públicas y otras instancias sociales y académicas -organizaciones de la sociedad civil, docentes, directivos, madres y padres de familia y/o tutores- que conocieron, opinaron y respaldaron el sentido de pertinencia y la calidad de la nueva propuesta". En: ACUERDO número 592 ... Op. cit., p. 8.
} 


\begin{tabular}{|c|c|c|}
\hline $\begin{array}{c}\text { Competencia 1-b: } \\
\begin{array}{c}\text { Capacidad de usar este conocimiento e } \\
\text { información de manera interactiva }\end{array}\end{array}$ & $\begin{array}{c}\text { Competencia 2-b: } \\
\text { La habilidad de cooperar }\end{array}$ & $\begin{array}{c}\text { Competencia 3-b: } \\
\text { La habilidad de formar y conducir } \\
\text { planes de vida y proyectos } \\
\text { personales }\end{array}$ \\
\hline $\begin{array}{c}\text { Competencia 1-c: } \\
\text { La habilidad de usar la tecnología de } \\
\text { forma interactiva }\end{array}$ & $\begin{array}{c}\text { Competencia 2-c: } \\
\text { La habilidad de manejar y resolver conflictos }\end{array}$ & $\begin{array}{c}\text { Competencia 3-c: } \\
\text { La habilidad de afirmar derechos, } \\
\text { intereses, limites y necesidades }\end{array}$ \\
\hline
\end{tabular}

"Estas categorías, cada una con un enfoque específico, están interrelacionadas, y colectivamente, forman la base para identificar y mapear las competencias clave. La necesidad de que los individuos piensen y actúen reflexivamente es fundamental en este marco de competencias. La reflexión involucra no sólo la habilidad de aplicar de forma rutinaria una fórmula o método para confrontar una situación, también la capacidad de adaptarse al cambio, aprender de las experiencias y pensar y actuar con actitud crítica" (p. 4).

"Los individuos necesitan apoyarse en competencias clave que les permitan adaptarse a un mundo caracterizado por el cambio, la complejidad y la interdependencia. Estas competencias deben ser adecuadas para un mundo en donde:

- La tecnología cambia rápida y continuamente, y aprender a trabajar con ella no requiere dominio único de los procesos, sino también capacidad de adaptación.

- Las sociedades en su transformación son más diversas y fragmentadas, y las relaciones interpersonales requieren de mayor contacto con personas diferentes a uno.

- La globalización está creando nuevas formas de interdependencia y las acciones están sujetas tanto a influencias (como la competencia económica) y consecuencias (como la contaminación) que van más allá de la comunidad local o nacional del individuo" (p. 6).

Definiciones de competencias por campos de conocimientos del "Programe International for Student Assessment" (PISA):

Competencia en lectura: La capacidad de comprender, utilizar y reflexionar sobre textos escritos, para lograr sus propias metas, desarrollar su conocimiento y potencial, y participar en la sociedad.

Competencia en Matemáticas: La capacidad de identificar y comprender el rol que las matemáticas juegan en el mundo, hacer juicios bien fundamentados y usar y comprometerse con las matemáticas de formas que se logren satisfacer las necesidades de la vida propia como ciudadano constructivo, preocupado y reflexivo.

Competencia Científica: La capacidad de usar el conocimiento cientifico, identificar las cuestiones científicas y concluir con base en la evidencia para comprender y ayudar a tomar decisiones sobre el mundo natural y los cambios hechos a través de la actividad humana.

Este marco de referencia conceptual se aplica igualmente a las "competencias" que deben desarrollarse en la escuela y a aquellas que pueden ser desarrolladas a lo largo de la vida. Por esta razón, también proporciona un solo esquema para desarrollar evaluaciones escolares y evaluaciones para competencias de adultos. Parte central del concepto de "aprendizaje para la vida" es la afirmación de que "no todas las competencias que son relevantes para la vida", pueden ser proporcionadas por la Educación Básica, porque:

- Las competencias se desarrollan y cambian a lo largo de la vida, con la posibilidad de adquirir o "perder" competencias conforme se crece; 
- Las demandas sobre los individuos pueden cambiar a lo largo de sus vidas adultas como resultado de transformaciones en la tecnología y en las estructuras sociales y económicas; y

- La psicología del desarrollo muestra que el desarrollo de competencias no finaliza en la adolescencia, sino continúa a lo largo de los años adultos. En particular, la habilidad de pensar y actuar reflexivamente, que es parte central del marco, crece con la madurez.

Competencias y "perfil de egreso" para la Educación Básica en México (SEP)"

En el mundo contemporáneo cada vez son más altos los niveles educativos requeridos a hombres y mujeres para participar en la sociedad y resolver problemas de carácter práctico. En este contexto es necesaria una Educación Básica que contribuya al desarrollo de competencias amplias para mejorar la manera de vivir y convivir en una sociedad cada vez más compleja; por ejemplo, el uso eficiente de herramientas para pensar, como el lenguaje, la tecnología, los símbolos y el propio conocimiento; la capacidad de actuar en grupos heterogéneos y de manera autónoma.

La investigación educativa ha buscado precisar el término "competencias", coincidiendo en que éstas se encuentran estrechamente ligadas a conocimientos sólidos; ya que su realización implica la incorporación y la movilización de conocimientos específicos, por lo que no hay competencias sin conocimientos.

Una competencia implica un saber hacer (habilidades) con saber (conocimiento), así como la valoración de las consecuencias de ese hacer (valores y actitudes). En otras palabras, la manifestación de una competencia revela la puesta en práctica de conocimientos, habilidades, actitudes y valores para el logro de propósitos precisos en contextos y situaciones diversas, por esta razón se concibe a la "competencia" como la movilización de conocimientos (Perrenoud, 1999). Lograr que la Educación Básica contribuya a la formación de ciudadanos con estas características, implica plantear el desarrollo de competencias como propósito educativo central.

\section{El "perfil de egreso" de la Educación Básica en México}

El perfil de egreso de la Educación Básica, según la SEP (publicado en 2009), tiene un papel muy importante en el proceso de articulación de los tres niveles del subsistema (Preescolar, Primaria y Secundarial, que constituyen esta etapa de escolaridad obligatoria en México. Las razones de ser de dicho perfil son las siguientes:

\footnotetext{
"Secretaría de Educación Pública (SEP), Plan y Programas. Educación Primaria. México (2009) y Acuerdo Secretarial 592 por el que se establece la articulación de la Educación Básica (2011). 
2.1 Definir el tipo de estudiante que se espera formar a lo largo de la Educación Básica.

\subsection{Ser un referente común, tanto para la definición de los contenidos como para las orientaciones didácticas que guían el estudio de las asignaturas que forman el currículo.}

\subsection{Servir de base para valorar la eficacia del proceso educativo.}

El perfil de egreso plantea un conjunto de rasgos que los estudiantes deberán mostrar al término de la Educación Básica, como garantía de que podrán desenvolverse en cualquier ámbito en el que decidan continuar su desarrollo. Dichos rasgos son el resultado de una formación que destaca la necesidad de desarrollar competencias para la vida, que además de conocimientos y habilidades incluyen actitudes y valores para enfrentar con éxito diversas tareas.

El logro de los rasgos del perfil de egreso supone una tarea compartida entre los campos de conocimiento que integran los planes de estudio de la Educación Básica.

Los planes y programas de estudio de Preescolar, Primaria y Secundaria en México se han construido de manera articulada y con el principio general de que la escuela en su conjunto, y en particular los docentes, dirijan los aprendizajes de los alumnos mediante el planteamiento de desafíos intelectuales, el análisis y la socialización de lo que éstos producen, la consolidación de lo que se aprende y su utilización en nuevos desafíos para seguir aprendiendo. Así, el paso de los alumnos por la escolaridad básica se hará de manera coherente y sin traslapes o vacíos en las diversas líneas de estudio.

Como resultado del proceso de formación a lo largo de la escolaridad básica en México, el alumno mostrará los siguientes rasgos según la SEP (2009):

- Utiliza el lenguaje oral y escrito para comunicarse con claridad y fluidez e interactuar en distintos contextos sociales y culturales. Además, posee las herramientas básicas para comunicarse en una lengua adicional.

- Argumenta y razona al analizar situaciones, identifica problemas, formula preguntas, emite juicios, propone soluciones y toma decisiones. Valora los razonamientos y la evidencia proporcionada por otros y puede modificar, en consecuencia, los propios puntos de vista.

- Busca, selecciona, analiza, evalúa y utiliza la información proveniente de diversas fuentes.

- Interpreta y explica procesos sociales, económicos, financieros, culturales y naturales para tomar decisiones individuales o colectivas, en función del bien común.

- Conoce y ejerce los derechos humanos y los valores que favorecen la vida democrática, actúa y pugna por la responsabilidad social y el apego a la ley.

- Asume y practica la interculturalidad como riqueza y forma de convivencia en la diversidad social, étnica, cultural y lingüistica. 
- Conoce y valora sus características y potencialidades como ser humano; sabe trabajar en equipo; reconoce, respeta y aprecia la diversidad de capacidades en los otros, y emprende y se esfuerza por lograr proyectos personales o colectivos.

- Promueve y asume el cuidado de la salud y del ambiente, como condiciones que favorecen un estilo de vida activo y saludable.

- Aprovecha los recursos tecnológicos a su alcance, como medios para comunicarse, obtener información y construir conocimiento.

- Reconoce diversas manifestaciones del arte, aprecia la dimensión estética y es capaz de expresarse artísticamente.

\section{Las denominadas "competencias para la vida"}

La idea de "desarrollar competencias" moviliza y dirige todos estos componentes hacia la consecución de objetivos o metas concretos; son más que el saber, el saber hacer o el saber ser. Las competencias se manifiestan en la acción de manera integrada. Poseer sólo conocimientos o habilidades no significa ser competente: "se pueden conocer las reglas gramaticales, pero ser incapaz de redactar una carta; se pueden enumerar los derechos humanos y, sin embargo, discriminar a las personas con alguna discapacidad".

"La movilización de saberes (saber hacer con saber y con conciencia respecto del impacto de ese hacer) se manifiesta tanto en situaciones comunes de la vida diaria como en situaciones complejas y ayuda a visualizar un problema, poner en juego los conocimientos pertinentes para resolverlo, reestructurarlos en función de la situación, así como extrapolar o prever lo que hace falta. Algunos ejemplos de estas situaciones son: diseñar y aplicar una encuesta, organizar una actividad, escribir un cuento o un poema, editar un periódico. De estas experiencias se puede esperar una toma de conciencia de ciertas prácticas sociales y comprender, por ejemplo, que escribir un cuento no es sólo cuestión de inspiración, pues demanda trabajo, perseverancia y método" (RIEB, 2009).

Las competencias que aquí se proponen contribuirán al logro del perfil de egreso y deberán desarrollarse desde todas las asignaturas, procurando que se proporcionen oportunidades y experiencias de aprendizaje que sean significativas para todos los alumnos y alumnas ${ }^{12}$.

- Competencias para el aprendizaje permanente. Implican la posibilidad de aprender, asumir y dirigir el propio aprendizaje a lo largo de la vida, de integrarse a la cultura escrita, así como de movilizar los diversos saberes culturales, lingüisticos, sociales, científicos y tecnológicos para comprender la realidad.

- Competencias para el manejo de la información. Se relacionan con la búsqueda, identificación, evaluación, selección y sistematización de información; el pensar, reflexionar, argumentar y expresar juicios críticos; analizar, sintetizar, utilizar y compartir información; el conocimiento y manejo de distintas lógicas de construcción del conocimiento en diversas disciplinas y en los distintos ámbitos culturales.

\footnotetext{
${ }^{12}$ Secretaría de Educación Pública (SEP), Plan y Programas. Educación Primaria. México (2009) 
- Competencias para el manejo de situaciones. Son aquellas vinculadas con la posibilidad de organizar y diseñar proyectos de vida, considerando diversos aspectos, como los históricos, sociales, políticos, culturales, geográficos, ambientales, económicos, académicos y afectivos, y de tener iniciativa para llevarlos a cabo, administrar el tiempo, propiciar cambios y afrontar los que se presenten; tomar decisiones y asumir sus consecuencias, enfrentar el riesgo y la incertidumbre, plantear y llevar a buen término procedimientos o alternativas para la resolución de problemas, y manejar el fracaso y la desilusión.

- Competencias para la convivencia. Implican relacionarse armónicamente con otros y con la naturaleza; comunicarse con eficacia; trabajar en equipo; tomar acuerdos y negociar con otros; crecer con los demás; manejar armónicamente las relaciones personales y emocionales; desarrollar la identidad personal y social; reconocer y valorar los elementos de la diversidad étnica, cultural y lingüística que caracterizan a nuestro país, sensibilizándose y sintiéndose parte de ella a partir de reconocer las tradiciones de su comunidad, sus cambios personales y del mundo.

- Competencias para la vida en sociedad. Se refieren a la capacidad para decidir y actuar con juicio crítico frente a los valores y las normas sociales y culturales; proceder a favor de la democracia, la libertad, la paz, el respeto a la legalidad y a los derechos humanos; participar tomando en cuenta las implicaciones sociales del uso de la tecnología; participar, gestionar y desarrollar actividades que promuevan el desarrollo de las localidades, regiones, el país y el mundo; actuar con respeto ante la diversidad sociocultural; combatir la discriminación y el racismo, y manifestar una conciencia de pertenencia a su cultura, a su país y al mundo.

\section{El Acuerdo Secretarial 592 de la SEP ${ }^{13}$.}

"El Plan de estudios 2011. Educación Básica es el documento rector que define las competencias para la vida, el perfil de egreso, los Estándares Curriculares y los aprendizajes esperados que constituyen el trayecto formativo de los estudiantes, y que se propone contribuir a la formación del ciudadano democrático, crítico y creativo que requiere la sociedad mexicana en el siglo XXl, desde las dimensiones nacional y global, que consideran al ser humano y al ser universal". Art. 2º (D.O.F., 19 ago. 2011, Pp. 11).

\begin{tabular}{|c|c|c|c|c|c|}
\hline PERFIL DE EGRESO: & $\begin{array}{l}\text { COMPETENCIAS } \\
\text { PARA LA VIDA: }\end{array}$ & $\begin{array}{l}\text { ESTÁNDARES } \\
\text { CURRICULARES: }\end{array}$ & $\begin{array}{l}\text { APRENDIZAJES } \\
\text { ESPERADOS: }\end{array}$ & $\begin{array}{c}\text { PRINCIPIOS PEDAGÓGICOS } \\
\text { Y/O ASPECTOS } \\
\text { METODOLÓGICOS: }\end{array}$ & $\begin{array}{l}\text { NOTA TÉCNICA } \\
\text { ACLARATORIA: }\end{array}$ \\
\hline $\begin{array}{l}\text { El perfil de egreso } \\
\text { plantea rasgos } \\
\text { deseables que los } \\
\text { estudiantes deberán } \\
\text { mostrar al término de } \\
\text { la Educación Básica, } \\
\text { como garantía de que } \\
\text { podrán desenvolverse } \\
\text { satisfactoriamente en } \\
\text { cualquier ámbito en el } \\
\text { que decidan continuar } \\
\text { su desarrollo. Dichos } \\
\text { rasgos son el resultado } \\
\text { de una formación que }\end{array}$ & $\begin{array}{l}\text { "Una competencia } \\
\text { es la capacidad de } \\
\text { responder a } \\
\text { diferentes } \\
\text { situaciones, e } \\
\text { implica un saber } \\
\text { hacer (habilidades) } \\
\text { con saber } \\
\text { (conocimientol, así } \\
\text { como la valoración } \\
\text { de las } \\
\text { consecuencias de } \\
\text { ese hacer (valores y } \\
\text { actitudes)". Art. } 2^{\circ} \text {. }\end{array}$ & $\begin{array}{l}\text { "Los Estándares } \\
\text { Curriculares son } \\
\text { descriptores de logro } \\
\text { y definen aquello que } \\
\text { los alumnos } \\
\text { demostrarán al } \\
\text { concluir un periodo } \\
\text { escolar; sintetizan los } \\
\text { aprendizajes } \\
\text { esperados que, en } \\
\text { los programas de } \\
\text { Educación Primaria y } \\
\text { Secundaria, se } \\
\text { organizan por }\end{array}$ & $\begin{array}{l}\text { "Los } \\
\text { aprendizajes } \\
\text { esperados son } \\
\text { indicadores de } \\
\text { logro que, en } \\
\text { términos de la } \\
\text { temporalidad } \\
\text { establecida en } \\
\text { los programas } \\
\text { de estudio, } \\
\text { definen lo que } \\
\text { se espera de } \\
\text { cada alumno en } \\
\text { términos de }\end{array}$ & $\begin{array}{l}\text { I.1. Centrar la atención en } \\
\text { los estudiantes y en sus } \\
\text { procesos de aprendizaje. } \\
\text { I.2. Planificar para } \\
\text { potenciar el aprendizaje. } \\
\text { I.3. Generar ambientes de } \\
\text { aprendizaje. } \\
\text { I.4. Trabajar en } \\
\text { colaboración para construir } \\
\text { el aprendizaje. I.5. Poner } \\
\text { énfasis en el desarrollo de } \\
\text { competencias, el logro de } \\
\text { los Estándares Curriculares } \\
\text { y los aprendizajes }\end{array}$ & $\begin{array}{l}\text { "...el aprendizaje } \\
\text { de cada alumno y } \\
\text { del grupo se } \\
\text { enriquece en y } \\
\text { con la interacción } \\
\text { social y cultural, } \\
\text { con retos } \\
\text { intelectuales, } \\
\text { sociales, afectivos } \\
\text { y físicos, y en un } \\
\text { ambiente de } \\
\text { trabajo respetuoso y } \\
\text { colaborativo". Art. }\end{array}$ \\
\hline
\end{tabular}

\footnotetext{
${ }^{13}$ Publicado en el Diario Oficial de la Federaciōn el 19 de agosto de 2011, Secretaría de Educación Pública, México.
} 


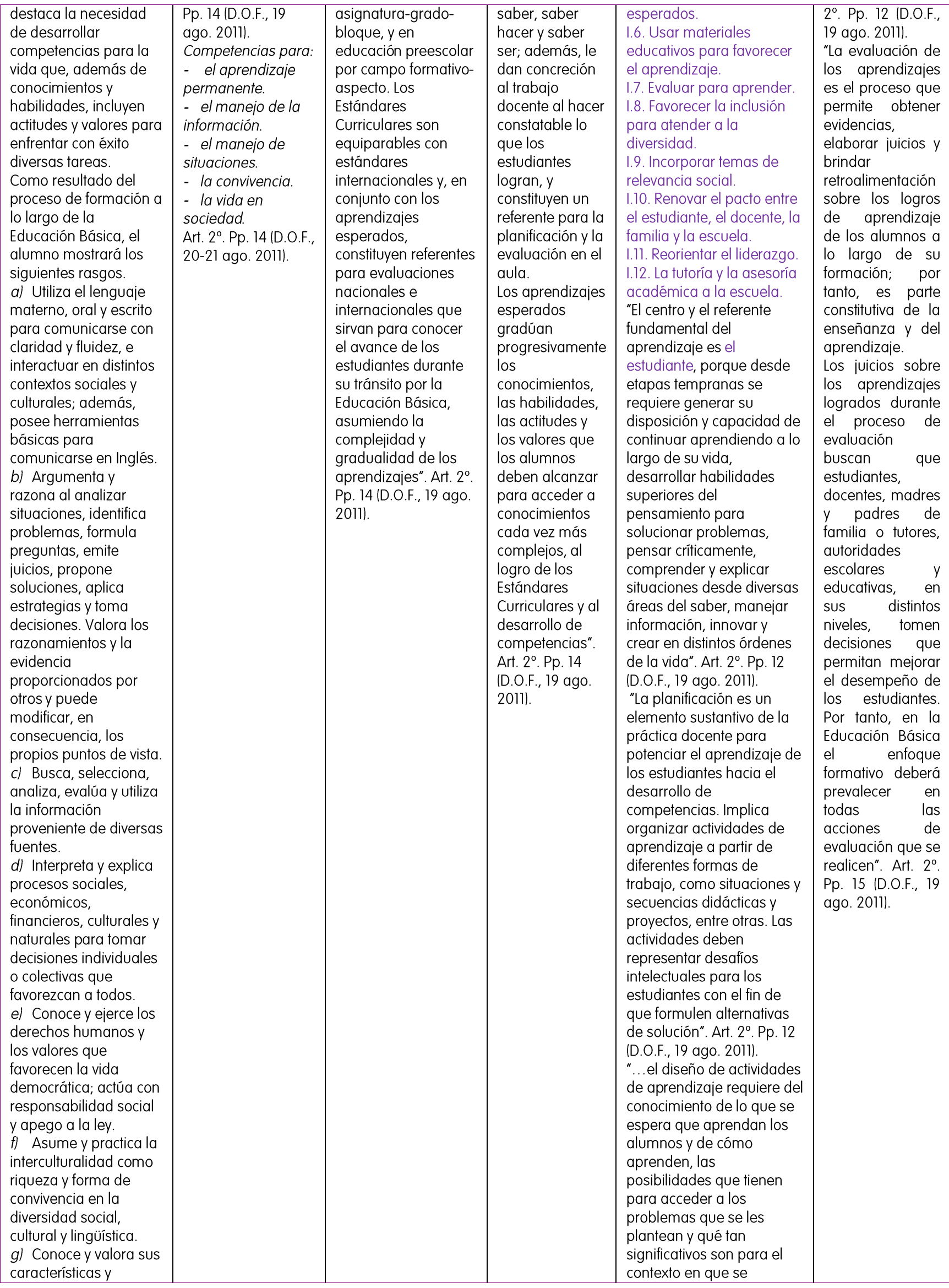




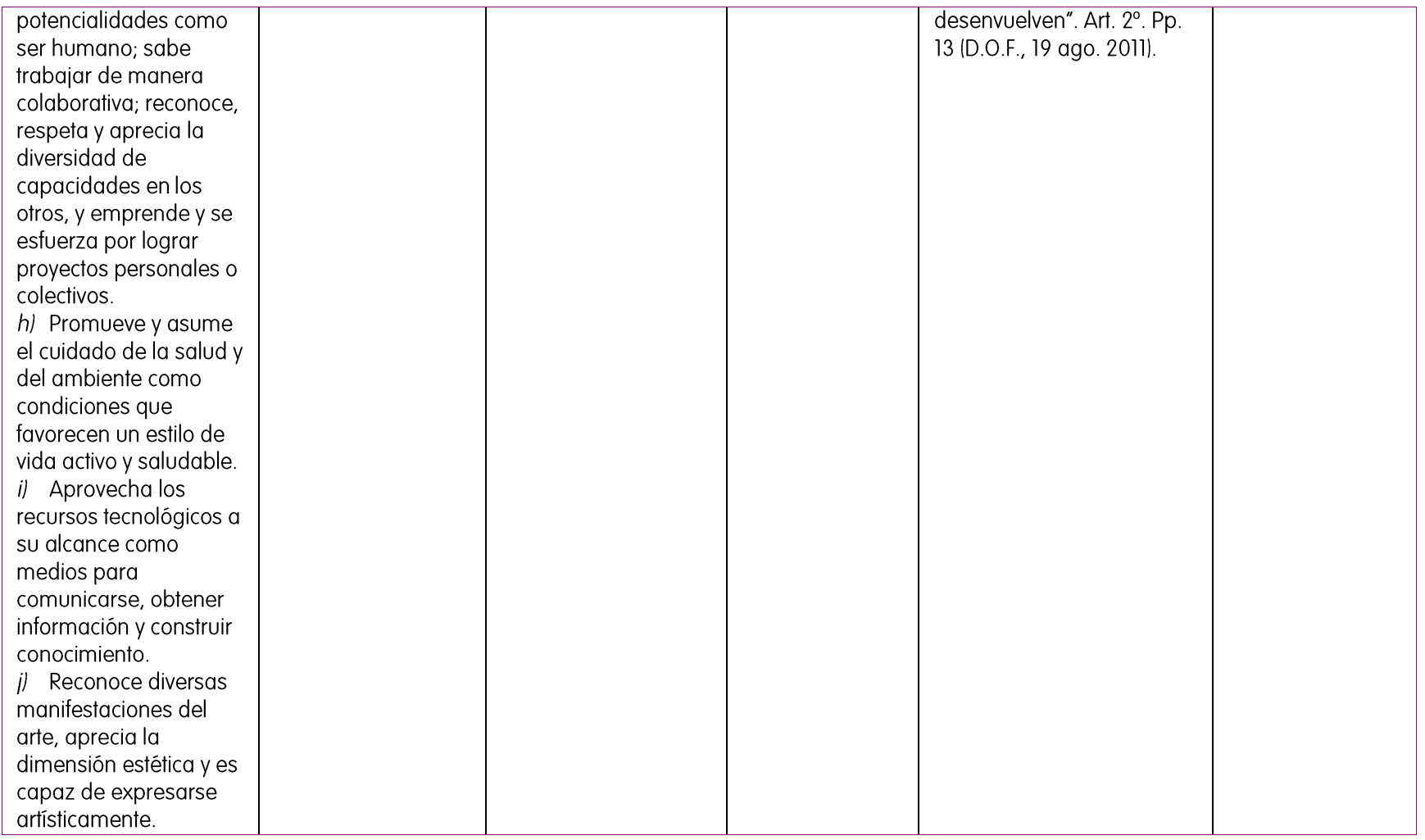

\section{El Pensamiento estratégico y el aprendizaje autónomo como alternativas: hacia la búsqueda de nuevos criterios y un lenguaje diferente}

En un esfuerzo por conciliar al diseño curricular basado "en el desarrollo de competencias educativas" con un enfoque más completo y menos limitado lel enfoque centrado en el Pensamiento Estratégico y el Aprendizaje Autónomo ${ }^{14}$ ), en la sección que a continuación se presenta se describen algunos ejemplos de aprendizajes esperados durante el desarrollo del alumno de Educación Primaria por ciclo trianual $\left(1^{\circ}\right.$., $2^{\circ}$. y $3^{\circ}$. grados constituyen la etapa o periodo $\mathrm{I}$; mientras que $4^{\circ}$., $5^{\circ}$. y $6^{\circ}$. grados, la etapa $\mathrm{O}$ periodo III, mediante una caracterización, no sólo marcada por criterios anclados en las denominadas "competencias clave", sino por criterios fundados en las dinámicas del "saber cómo", el "saber por qué", "saber para qué" y en el "saber por cuenta propia".

Propuesta general de "aprendizajes genéricos esperados" durante y al término de la Educación Primaria, por etapa trianual (Incluye campos de conocimientos, habilidades psicomotrices y actitudesvalores):

\footnotetext{
${ }^{14}$ Una Pedagogía basada en el Pensamiento Estratégico de los alumnos es una conjugación de reflexiones y planteamientos teóricos y metodológicos en torno a los procesos de desarrollo de los saberes estratégicos ("saber cómo y por qué") y saberes autónomos ("saber por cuenta propia"), que pretenden ir más allá de los objetivos conductuales de B. Bloom, cuya característica esencial es la ejecución de actividades didácticas a través de asociaciones entre el "ambiente" y la "conducta"; así como más allá del enfoque por "objetivos o propósitos de aprendizaje", que tiene una fuerte carga directiva, generalmente centrada en la labor del docente ("enseñanza"), y que ubica en un lugar periférico a las actividades de aprendizaje del alumno.
} 
a) Educación Primaria. Ełapa o periodo I: Primero, segundo y tercer grados.

Aprendizajes genéricos en el campo del Pensamiento Estratégico, en términos de "saber cómo", "saber por qué", "saber para qué" y "saber por cuenta propia":

Al concluir el tercer grado de Educación Primaria, el alumno: Desarrollará algunas habilidades clave en los ámbitos del lenguaje y la comunicación (orales y escritas), a efecto de intercambiar ideas, mensajes y puntos de vista; también desarrollará algunos estilos de interacción en los ámbitos del desarrollo personal y social, con la finalidad de lograr una adecuada relación con los otros; consolidará el pensamiento matemático, a través del manejo de nociones, procedimientos básicos y la comprensión de principios sobre el sentido numérico, y para resolver problemas de formas, espacios y medidas, y manejo de la información relacionados con prácticas sociales; se iniciará en el ejercicio de actitudes hacia la exploración y conocimiento del mundo, para comprender el entorno tanto natural como social, y actuar a favor de su preservación o armonía; tendrá gusto por la expresión artística, como medios para el desarrollo de la persona y el equilibrio de la subjetividad y la creatividad; además, identificará a la familia, sus hábitos y costumbres, asi como sus tradiciones y valores, tanto sociales como culturales, con la intención de adaptarse de manera positiva en ese ámbito. Finalmente, desarrollará las capacidades para socializar e integrarse, asi como para trabajar en equipo, con la finalidad de valorar las ventajas y beneficios que trae consigo estas actitudes y aptitudes individuales y colectivas.

b) Educación Primaria. Ełapa o periodo II: Cuarto, quinto y sexto grados.

Al terminar el sexto grado: Los alumnos mostrarán dominio de las habilidades en los ámbitos del lenguaje y la comunicación (orales y escritas); así como aprovechará las condiciones y los medios para lograr su desarrollo personal y social, en contextos sociales de mutuo respeto y autonomía ante sus compañeros, maestros y autoridades; ejercitará el pensamiento matemático tanto en el uso de herramientas propias de este campo del conocimiento como en el planteamiento y solución de problemas sobre el sentido numérico y pensamiento algebraico, asi como de formas, espacios y medidas, y manejo de la información; presentará actitudes y habilidades hacia la exploración y conocimiento del mundo, a través del diseño, desarrollo y evaluación de proyectos de aprendizaje en ciencias y otras evidencias relacionadas con ese campo formativo; tendrá gusto por la expresión artística como un medio seguro para ejercer la creatividad y la libertad de pensamiento; además, dominará los conocimientos sobre la familia, sus hábitos y costumbres, así como sus tradiciones, códigos y valores éticos, tanto personales como sociales y culturales, a efecto de llevar a cabo una relación equilibrada con los demás. Ejercitará y contribuirá al desarrollo de capacidades para socializar e integrarse en distintos contextos sociales, así como para colaborar en equipo de manera productiva, propositiva y autónoma.

En otras palabras, al término de la Educación Primaria y en el contexto de estos ejemplos, el alumno: Empleará el lenguaje escrito y oral con fluidez y de manera comprensiva; analizará situaciones, planeará y solucionará problemas tanto del ámbito de las Matemáticas como de las Ciencias en general; expresará sus ideas con base en argumentos; será capaz de interpretar y opinar en torno a procesos naturales, sociales e históricos; identificará, discutirá y ejercerá los derechos humanos y los valores ciudadanos lequidad de género, democracia, igualdad, transparencia, desarrollo sustentable, responsabilidad social, entre otros), en un marco de confianza, equidad e inclusión educativas; presentará actitudes científicas de curiosidad y creatividad, así como de búsqueda autónoma de información y cuestionamiento de teorías o enfoques sobre los fenómenos naturales, tanto de su persona como de su 
entorno; aplicará el pensamiento científico, humanístico y tecnológico de manera crítica y reflexiva ante fenómenos naturales y sociales de su entorno y universales; apreciará la expresiones artísticas y establecerá relaciones sociales con base en un desarrollo personal y social equilibrado y adecuado a su edad. Dominará y desarrollará capacidades para socializar e integrarse en distintos contextos sociales, así como para trabajar en equipo de manera productiva, propositiva y autónoma, tanto con personas de su edad como con personas mayores.

\section{Conclusiones}

1. El modelo que ha servido como fundamento para emprender la Reforma Curricular actual, (20092011), de la Educación Básica en México, es el denominado modelo de "desarrollo de competencias o para la vida", el cual no es el único existente en la literatura moderna de la investigación educativa relativa al diseño curricular (un enfoque alternativo, por ejemplo, que se ha manejado en Italia, es el modelo llamado "histórico").

2. Cuando la estructura y los argumentos de un modelo de planeación que pretende fundamentar una reforma curricular nacional de la Educación Básica, se someten de manera centralizada al examen de los especialistas e investigadores pares en diseño y análisis curricular, y de la sociedad civil en general, y dicho modelo se convierte en norma establecida, como es el caso que se menciona en este ensayo, éste pierde una parte importante de su legitimidad académica y su consistencia técnica. Una situación como ésta genera importantes resistencias, en términos de la actuación de los agentes educativos que operan o hacia los que está destinada dicha reforma: Docentes, alumnos, directivos, asesores técnicos, personal de apoyo y sociedad en general.

3. Por lo anterior, es de suma importancia dedicar todos los esfuerzos institucionales que sean necesarios y pertinentes, para que las figuras centrales del cambio y la mejora, es decir, los docentes, reconozcan la estructura y los argumentos de la Reforma en cuestión, a través de procesos de capacitación, actualización y profesionalización continua o permanente, en un clima de mediación y consenso, que permitan a su vez una adecuada preparación académica y actitudinal, como requisitos indispensables para transformar las prácticas educativas y didácticas y, en consecuencia, mejorar los aprendizajes escolares.

4. Dichos procesos deberán orientarse hacia la transformación tanto de la práctica docente como de la operatividad didáctica esperada, desde el nuevo diseño curricular, y como parte sustantiva del perfil de profesionalización definido en torno a las funciones, responsabilidades compartidas y efectos o impactos sociales e individuales de la enseñanza.

5. Si bien existe una sistematización y reorganización de la taxonomía de los "paquetes" de aprendizajes, a partir del enfoque centrado en el (la) alumno(a) para "desarrollar en él o ella las competencias educativas", el cual está definido en el nuevo currículum escolar, la sugerencia de expertos de la OCDE y adoptado por los gobiernos de los países miembros), dicho modelo presenta algunas limitaciones en sus argumentos, a saber: "Fallas de origen"; excesivas generalización y transferencia acerca de lo que se espera de los alumnos entre distintos niveles educativos; contradicciones en la selección de contenidos; fuerte énfasis en el "pragmatismo", entre otros. 
6. En Educación Básica se requiere ir más allá del enfoque o modelo basado en el "desarrollo de competencias educativas", sobre todo cuando éste se encuentra atrapado entre el pragmatismo ("saber hacer") y el uso mecánico de procedimientos ("saber cómo"), o se limita simplemente a promover estrategias que sólo buscan que el sujeto "resuelva problemas prácticos" o "inmediatos".

7. Un modelo de Reforma curricular para la Educación Básica alternativo, habrá de considerar y superar las inconsistencias o limitaciones del esquema "decretado", a efecto de buscar nuevas vías u orientaciones, a través del desarrollo de esquemas flexibles y centrados en el alumno: para que éste "sepa hacer", pero que también se pregunte: ¿"para qué" hago eso? ¿"qué sentido tiene..."?; que el alumno "sepa cómo hacer o cómo desarrollar una actividad", pero que comprenda los procedimientos y logre distinguir entre distintos tipos de éstos, y sepa también elegir estrategias adecuadas para solucionar problemas en un sentido amplio, no coyuntural. Para ello el sujeto educativo requiere desarrollar el pensamiento estratégico ("saber cómo y por qué"), asî como los principios del aprendizaje autónomo ("aprender por cuenta propia").

8. A partir de estos criterios sería conveniente e interesante desarrollar, en el aula y en la escuela de Educación Básica en general, nuevas rutas críticas para diseñar, aplicar y evaluar situaciones o ambientes a favor del desarrollo de los aprendizajes escolares y para la vida.

\section{Referencias bibliográficas:}

Araujo, J.B. y C.B. Chadwick (1988). Tecnología educacional. Paidós (educador). Barcelona, España.

Bloom, Benjamin, S. (1978) Taxonomía de los objetivos de la educación. El Ateneo. Argentina. (Tomos I y III.

Coll, César. Las competencias en la educación escolar: algo más que una moda y mucho menos que un remedio. Revista Innovación Educativa, No. 161 (s/f).

Coll, César y Elena Martín (2006). Vigencia del debate curricular. Aprendizajes básicos, competencias y estándares. II Reunión del Comité Intergubernamental del Proyecto Regional de Educación para América Latina y el Caribe (PRELAC). UNESCO-OREALC. Oficina Regional de Educación para América Latina y el Caribe. Santiago de Chile.

DAVIS, R.H.; L.T. AleXANDeR y S. Yelon (1988). Diseño de sistemas de aprendizaje. Un enfoque del mejoramiento de la instrucción. Trillas, 2a. edición. México.

GAGNÉ, Robert M. (1977) The conditions of learning. Holt, Rinehart and Winston. New York.

GaRAGORRI, Xabier. "Currículo basado en competencias: aproximación al estado de la cuestión" (Revista Innovación Educativa, No. 161, s/f).

MAGER, R. (1975) La confección de objetivos para la enseñanza. Ministerio de Educación. MINED (sin lugar de publicación).

Organización para la CoOPeración y el DesarRollo EConómico (OCDE, 2001). La definición y selección de Competencias Clave. Resumen ejecutivo preparado por el grupo educativo de la OCDE y traducido con fondos de la Agencia de los Estados Unidos para el Desarrollo Internacional (USAID).

Secretaría de Educación Pública (SEP, 2009). Plan y Programas. Educación Básica. Educación Primaria. México.

SECRETARÍA de EdUCACIÓN PúBLICA (SEP, 2011). Acuerdo secretarial 592 por el que se establece la articulación de la Educación Básica. Diario Oficial de la Federación, 19 de agosto de 2011. 